\title{
Téoros
}

Revue de recherche en tourisme

\section{Le site touristique aménagé des Gorges de la Fou en Pyrénées françaises}

\section{Commercialisation, médiation scientifique ou animation} culturelle et controverse paysagère

\section{André Suchet}

Volume 29, numéro 2, 2010

Géotourisme

URI : https://id.erudit.org/iderudit/1024870ar

DOI : https://doi.org/10.7202/1024870ar

Aller au sommaire du numéro

Éditeur(s)

Université du Québec à Montréal

ISSN

0712-8657 (imprimé)

1923-2705 (numérique)

Découvrir la revue

Citer cet article

Suchet, A. (2010). Le site touristique aménagé des Gorges de la Fou en Pyrénées françaises : commercialisation, médiation scientifique ou animation culturelle et controverse paysagère. Téoros, 29(2), 44-54.

https://doi.org/10.7202/1024870ar
Résumé de l'article

Ce travail propose une étude géographique et ethnographique du site des Gorges de la Fou : un canyon des Pyrénées orientales en France mis en tourisme par un ensemble d'aménagements (escaliers, passerelles, filet métallique de protection...) qui permettent la visite sécurisée pour tous les publics. Cet aménagement lourd du milieu naturel suscite une controverse paysagère. L'article présente le fonctionnement du site, les acteurs locaux du tourisme et la fréquentation (environ 80000 visiteurs par an). L'étude met en évidence la non-médiation scientifique au profit d'une animation culturelle des Gorges, puis l'activation de ce géomorphosite en ressource territoriale. 


\title{
Le site touristique aménagé des Gorges de la Fou en Pyrénées françaises Commercialisation, médiation scientifique ou animation culturelle et controverse paysagère
}

\author{
André SUCHET \\ Chargé d'enseignement \\ Institut de géographie alpine, Université de Grenoble/CNRS, Laboratoire PACTE \\ a.suchet@wanadoo.fr
}

\begin{abstract}
RÉSUMÉ: Ce travail propose une étude géographique et ethnographique du site des Gorges de la Fou : un canyon des Pyrénées orientales en France mis en tourisme par un ensemble d'aménagements (escaliers, passerelles, filet métallique de protection...) qui permettent la visite sécurisée pour tous les publics. Cet aménagement lourd du milieu naturel suscite une controverse paysagère. L'article présente le fonctionnement du site, les acteurs locaux du tourisme et la fréquentation (environ 80000 visiteurs par an). L'étude met en évidence la non-médiation scientifique au profit d'une animation culturelle des Gorges, puis l'activation de ce géomorphosite en ressource territoriale.
\end{abstract}

Mots-clés: Canyon, géomorphosite, aménagement, paysage, médiation scientifique.

Le tourisme associé aux géomorphosites, au sein de la catégorie plus vaste du géotourisme, doit beaucoup au tourisme souterrain. Les grottes touristiques, c'est-à-dire aménagées avec des passerelles en fer, des escaliers pour descendre ou remonter entre les salles et un bâtiment d'accueil à l'entrée qui abrite la billetterie ou un magasin de souvenirs, sont un secteur reconnu de l'industrie des loisirs et du tourisme en France. Le tourisme souterrain et l'aménagement des grottes n'est pas récent (Biot, 2006; Nicod, 1998; Schut, 2007). Selon Gauchon (1997) «les grottes et les gouffres [...] ont été mis en valeur avec une diversité et une ingéniosité étonnantes. Le tourisme souterrain apparaît comme l'une des formes les plus anciennes du tourisme en montagne». Plusieurs études montrent ainsi les dynamiques de développement puis de crise du tourisme souterrain (Biot et Gauchon, 2005; Gauchon, 1996). Cette centration sur le milieu souterrain laisse dans l'ombre d'autres géomorphosites aménagés suivant le même principe, à savoir les gorges ou canyons et cascades «remarquables» auxquels le public accède grâce à un système de passerelles et d'escaliers. Il peut s'agir d'une chute d'eau ou d'un point particulier dans un canyon, pour lequel un belvédère a été aménagé. D’autres fois, un parcours entier permet de suivre la gorge (système de passerelles au-dessus de l'eau, grilles, parapets...). Ces aménagements sont parfois situés sur l'un ou l'autre côté des berges, implantés plus ou moins discrètement dans la falaise.
Le présent article propose une étude géographique et ethnographique du site des Gorges de la Fou, un canyon des Pyrénées orientales en France mis en tourisme par un ensemble d'aménagements de ce type (voir illustrations 1, 6 et 7) et qui s'annonce être "un cas géologique unique et étonnant» dont l'importance en ferait «le site géologique le plus visité du département» (dépliant publicitaire et site internet de promotion). Les autres sites géomorphologiques mis en tourisme dans le département sont des grottes (Fontrabiouse, la Cova Bastera, les Canalettes, les Grandes Canalettes) et un ensemble de cheminées de fée localement nommé les Orgues d'Ille-sur-Têt (voir à ce sujet Lemartinel, 1998). Le département des Pyrénées-Orientales, partagé entre mer et montagne (neuf stations de ski en moyenne montagne, cinq stations thermales, 10 stations balnéaires dont Collioure et Argelès-sur-Mer), accueille chaque année quatre millions de touristes et quatre millions d'excursionnistes (ODT Pyrénées-Orientales, 2008; Peypoch et Solonandrasana, 2006). Situées en vallée du Tech, dans le Haut Vallespir, les Gorges de la Fou se trouvent entre le massif du Canigou au nord, et les crêtes frontières avec l'Espagne au sud, du col d'Ares au Roc de France. Avec 75454 visiteurs en 2009, il s'agit de l'un des principaux canyons aménagés pour la visite en France. Notons parmi les plus connus des autres sites touristiques du même type les Gorges du Pont du Diable, les Gorges du Fier ou les Gorges de la Diosaz dans les Alpes du Nord et les Gorges de Kakuetta dans les 
Pyrénées occidentales. Dans le cas des Gorges de la Fou, il s'agit de mettre à jour les éléments du système touristique local en jeu dans la production du site (en référence au cadre de l'offre et de la demande; Chadefaud, 1988; Clary, 1993), puis d'étudier la controverse dont les aménagements du milieu sont l'objet. Dans le même temps, une étude ethnographique et géographique du tourisme tel qu'il est pratiqué dans les Gorges (référence aux études de Knafou et al., 1997; MIT, 2002) permet d'apporter contribution à l'étude des relations entre médiation scientifique (anciennement nommée vulgarisation), animation culturelle et commercialisation touristique d'un géomorphosite.

\section{Les Gorges de la Fou : un site touristique}

Les Gorges de la Fou sont un canyon au sens géomorphologique, c'est-à-dire un creusement lié à l'eau dans une roche devenant encaissante (Choppy, 1985 : 14; Palmer, 2007 : 408). L'hydroformation mécanique et/ou chimique crée une sorte de tuyau dans la masse rocheuse dont les parois deviennent plus ou moins hautes (canyon vient de l'espagnol cañón qui signifie tuyau). Dans les Gorges de la Fou, la hauteur des falaises atteint par moment 205 mètres. La longueur de roche creusée en canyon est de 1740 mètres, dont 1500 ont été aménagés de passerelles et d'escaliers. L'ensemble est protégé de filets métalliques pour prévenir d'éventuelles chutes de pierres (voir illustrations 6 et 7). Pendant le trajet, à 850 et 1000 mètres du début, deux grandes plateformes établissent une aire de repos avec téléphone de sécurité. Le toponyme «Fou» vient de l'ancien catalan fou qui désigne un défilé, un cirque étroit (Carreras-Marti et Giralt-Radigales, 1993 : 957).

Les documents touristiques des Gorges de la Fou expliquent que «longtemps [...] nul être humain n'osa s'aventurer dans ces sombres défilés», puis racontent "les exploits d'un Tarzan local, Père Besti, qui, au prix d'une gymnastique dont on évalue le péril, allait dénicher les aigles [...] En 1928, un groupe de cinq habitants d'Arles-sur-Tech et d'Amélie-lesBains entreprit pour la première fois la traversée totale des gorges. Cette exploration réunissait : Peperty, De Viaris, Soule, Riondet et Pujade, véritables pionniers du tourisme» (extrait de la feuille-guide de visite distribuée à l'entrée des gorges). Dans les documents, à chaque fois, l'histoire de ces personnes est déconsidérée : «un tarzan local», ou alors intégrée à l'univers du tourisme "véritables pionniers du tourisme». Le discours contribue ici à une subversion du lieu (au sens de l'équipe MIT, $2002: 221$ ), c'est-à-dire à un détournement de la fonction initiale de l'espace. Dans le cas des Gorges de la Fou, les usages traditionnels (la chasse aux rapaces, la pêche), les usages sportifs (la spéléologie, la descente en canyoning) ou l'activité scientifique sont ainsi volontairement oubliés.

Avec la construction d'une passerelle en bois en 1953, le site devient une visite payante sous l'initiative du maire d'Arles-sur-Tech, Baptiste Pams. L'idée vient d'un commerçant d'Arles-sur-Tech, François Pujade, montagnard local et membre de l'équipée dans les Gorges en 1928 :

À l'époque [...], chaque fois que quelqu'un cherchait une source ou un refuge dans le massif du Canigou, il devait faire le détour dans sa boutique. Car François connaissait tout [...]. C'était un homme capable de te dire : tiens, cette pierre n'était pas là l'année dernière ou il y a une

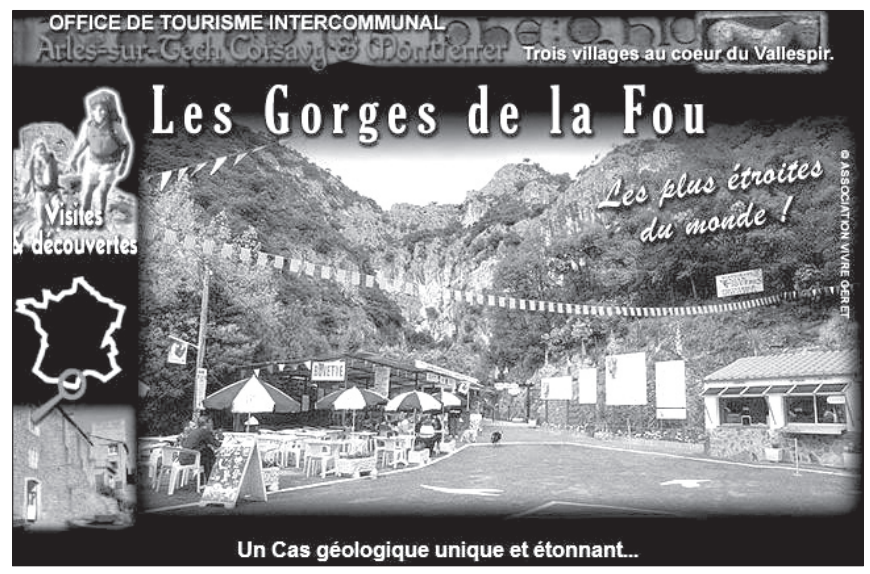

ILLUSTRATION 1 : Présentation commerciale de l'entrée des Gorges de la Fou (source : page d'accueil du site internet des Gorges de la Fou).

semaine [...] Il a encouragé la mairie d'Arles à exploiter ce site. (André Vinas, entretien cité par Paul Palau, Bernard Rieu et Patrice Teisseire-Dufour, 2009 : 68).

Dans les années 1950, François Pujade, prend la direction du syndicat d'initiative d'Arles-sur-Tech et devient promoteur du tourisme dans le département. Puis, en 1989 s'ouvre un Syndicat intercommunal à vocation unique pour l'exploitation des Gorges de la Fou (le SIVU des Gorges de la Fou). Il regroupe les communes d'Arles-sur-Tech, de Corsavy et de Montferrer. Effectivement, le tracé des Gorges concerne les trois communes. Le président actuel du site détaille :

L'idée, les fondateurs... ils venaient d'Arles-sur-Tech. Puis c'est Montferrer qui revendique, le maire était Madeleine Denamiel, et du coup, au dernier moment Corsavy aussi, car il en avait plus. À l'époque, le maire de Corsavy était Jean Vaills. En fait, la rive droite c'est Montferrer et tout en bas, un peu Arles mais très peu... très peu. Eux c'était plus l'antériorité. Tandis que toute la rive gauche c'est Corsavy. Donc finalement le SIVU sur les trois communes (Antoine Chrysostome, actuel président du SIVU des Gorges de la Fou, maire de Corsavy, entretien de 2010).

Cette affaire de concurrence entre initiative d'exploitation et propriété foncière ou communale du cours d'eau rappelle les conflits dans la production de l'espace thermal en Pyrénées (Chadefaud, 1988).

Actuellement, le SIVU des Gorges de la Fou, six salariés, plus cinq emplois saisonniers, permet d'entretenir les équipements et d'animer le site. La présidence du SIVU tourne entre les trois communes, initialement par période de deux ans, puis désormais de six ans (en phase avec les mandats municipaux). Parmi les acteurs du tourisme de ce système local (au sens de Clary, 1993), la culture commerçante reste importante, avec pour actuel vice-président du SIVU un artisan boucher-charcutier de Corsavy en charge des Gorges de la Fou à la mairie de Corsavy, Roger Coll. À l'entrée des Gorges se trouvent quatre zones de stationnement gratuites aménagés dans la pente (100 places). Une structure privée de six ou sept salariés en saison offre buvette/restauration, stand de produits locaux et 


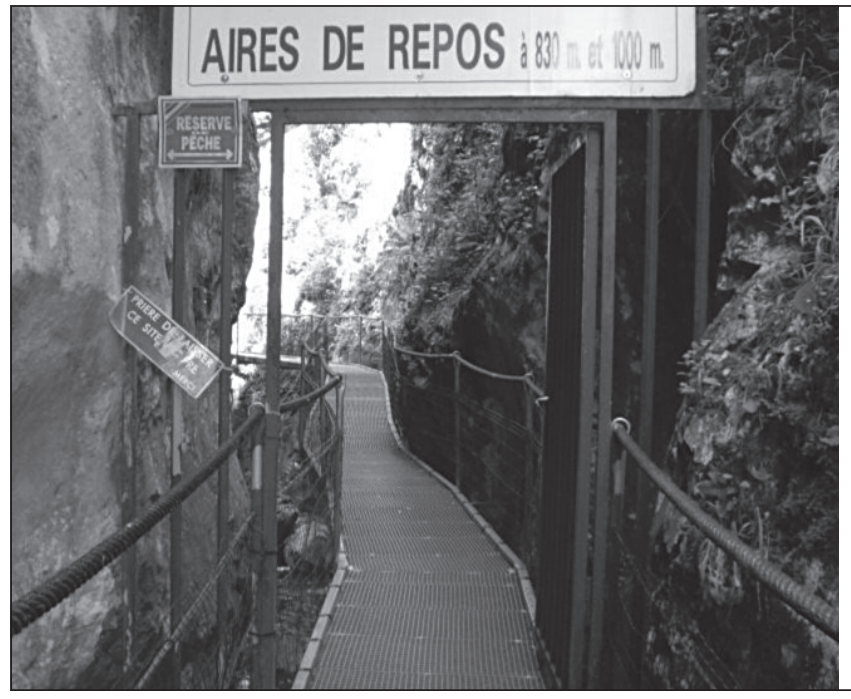

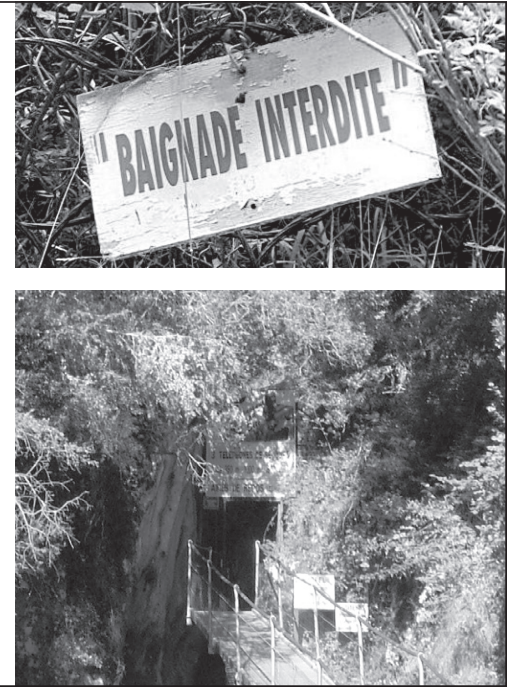

ILLUSTRATION 2 : Grille scellée dans la roche et panneaux d'entrée dans les Gorges de la Fou

(photos : André Suchet). de souvenirs (voir illustration 1). Un chenil gratuit permet de laisser son chien, qui n'est pas autorisé pendant la visite. Les Gorges de la Fou représentent un site touristique au sens de la typologie des lieux touristiques de l'équipe MIT (2002).

La subversion du lieu est totale avec un nombre impressionnant de panneaux d'interdictions à l'entrée du parcours (voir illustration 2). La pêche est interdite (zone réserve de pêche à la demande des municipalités), la baignade, et donc toute activité sportive, est également interdite. L'entrée, autorisée par une grille, n'est possible que de $10 \mathrm{~h}$ à $18 \mathrm{~h}$ du début avril à mi-novembre, de 9 h 30 à 18 h 30 pendant la pleine saison. Évidement plusieurs panneaux rappellent de «laisser propre ce site» et il est interdit de prélever une plante ou une roche. Comme le remarque le président du Comité départemental de spéléologie des Pyrénées-Orientales, Fabrice Fillols (entretien de 2010), les restrictions d'accès empêchent également la prospection spéléologique et donc la recherche scientifique. À aucun moment il n'est possible de quitter la passerelle. Indépendamment des mesures de sécurité (rambarde doublée d'un grillage, parapet) ou de la nécessité de circonscrire l'impact d'une fréquentation annuelle pouvant atteindre 100 000 passages (voir illustration 3), l'espace touristique demeure volontairement coupé de l'espace sauvage (voir illustrations 2 et 6). Par exemple, lorsqu'il ne s'agit pas d'un passage encaissé entre les falaises, un grillage avec fil de fer barbelé entoure le site, vraisemblablement pour éviter les visiteurs «clandestins». Pour aller plus loin, il faudrait comprendre les représentations de la nature introduites par cette séparation, ce conditionnement dans le temps et dans l'espace.

\section{Politique de commercialisation et fréquentation du site}

A priori, l'importance accordée au lieu interroge la possibilité d'une patrimonialisation de cet étroit paysage et de son environnement, c'est-à-dire l'établissement d'un patrimoine naturel des Gorges (tel qu'ont pu l'observer en d'autres espaces Duval, 2008, ou Reynard, 2005). Néanmoins, dans les faits, le lien peu évident entre le site des Gorges de la Fou et une mémoire locale, puis la modification controversée de l'intérieur du paysage-canyon éloignent d'une élaboration patrimoniale (Lazzarotti, 2003). Effectivement, si l'on considère ce géomorphosite pour sa valeur paysagère, les modifications de l'espace en escaliers, en passerelles et filet-auvent métallique (voir illustrations 6 et 7) contrarient la notion de protection contenue dans la définition d'une patrimonialisation de paysage. Au final, plutôt qu'un effet de patrimonialisation, les Gorges de la Fou ainsi exploitées semblent constituer une ressource territoriale en vallée du Tech, c'est-à-dire une spécificité locale activée par un projet (Gumuchian et Pecqueur, 2007).

Conjointement, alors même que les richesses géomorphologiques et la communication faite sur ce thème inciteraient à renforcer la médiation scientifique, les projets concernent explicitement une dimension culturelle. Le secrétaire du SIVU des Gorges de la Fou et adjoint administratif à la mairie de Corsavy témoigne : "Aujourd'hui, les discussions, les projets, c'est de profiter du site pour faire des manifestations culturelles, des spectacles» (Claude Couchevellou, entretien de 2010). Le président du SIVU et maire de Corsavy propose de «développer des animations, des spectacles sur le parking, des expos...» (Antoine Chrysostome, entretien de 2010). La voie du tourisme sportif en montagne, c'est-à-dire l'équipement d'un site d'escalade, la mise en réseau avec les moniteurs de canyoning qui encadrent l'activité dans plusieurs sites à proximité, l'indication des sites de décollage possibles en vol libre, voire l'aménagement d'une via ferrata... semble aussi fortement délaissée.

Une orientation relativement surprenante étant donné la concurrence de la côte déjà surchargée en spectacles, expositions et concerts. Pour le président du SIVU, les festivals et animations permettraient de «rallonger la période de fréquentation [...] pour les saisons creuses, parce qu'on ne fera pas plus en juillet/août. On est au maximum, il y a des limites physiques, des limites de sécurité. [Pendant certaines de nos visites pour observation, la passerelle était surchargée de monde à tel point qu'il devenait difficile d'avancer.] » (Antoine Chrysostome, entretien de 2010). La politique actuelle dans les 
ILLUSTRATION 3 : Fréquentation des Gorges de la Fou sur la période 1990-2009

(en nombre de visiteurs)

(la courbe supérieure comporte une correction d'anomalie : 5 mois de travaux en début de saison 1997 et 1998) (source : SIVU des Gorges de la Fou).

ILLUSTRATION 4 : Variations saisonnières de la fréquentation (en nombre de visiteurs) (compilation de données pour les années 1990, 1994, 1996 et 2001) (source : SIVU des Gorges de la Fou).
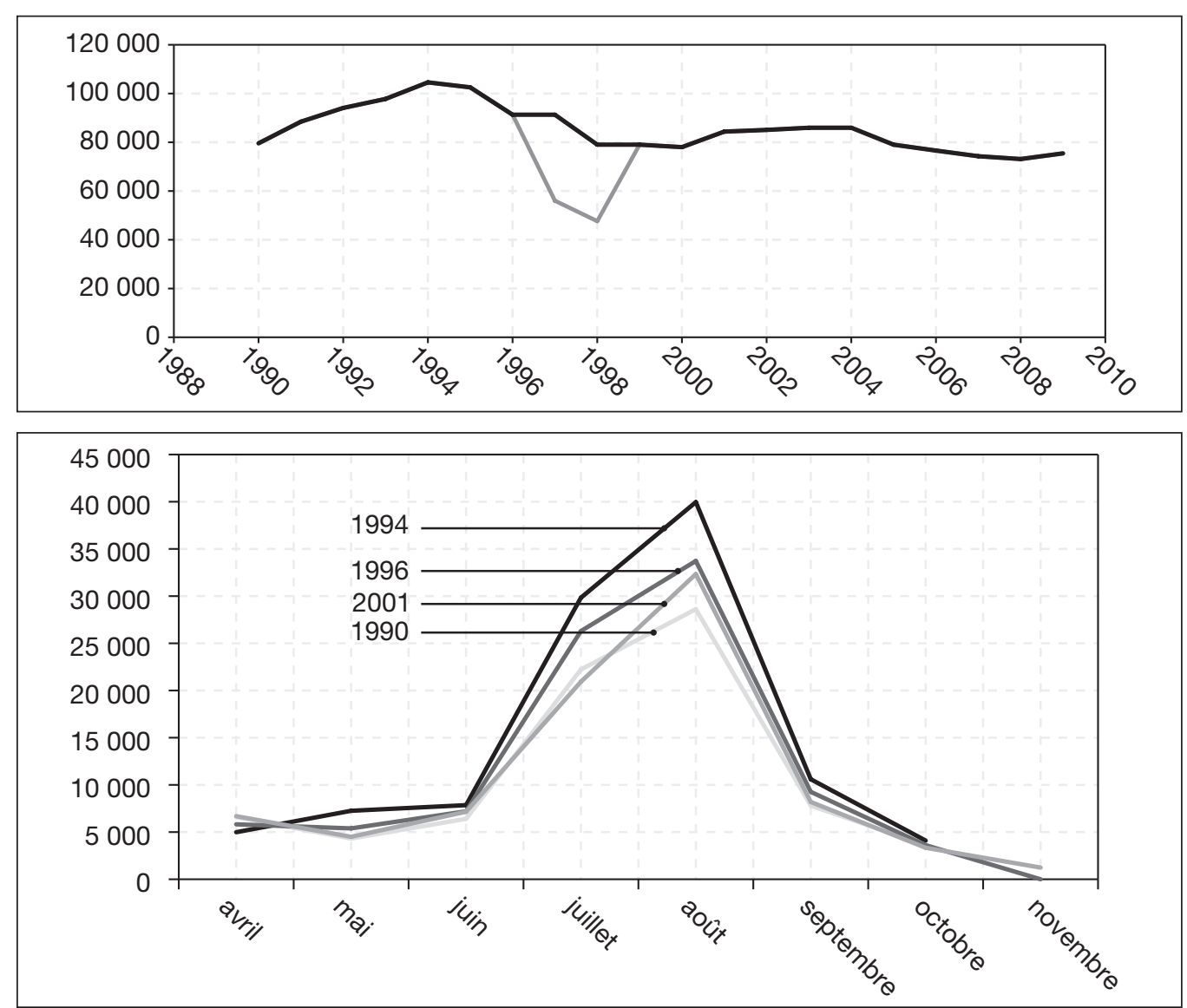

Gorges de la Fou se trouve en effet clairement quantitative. On retrouve la crainte d'un manque de fréquentation déjà observé dans les petites communes des Alpes du Nord à propos du ski, à chaque fois comme si le tourisme de masse était un exemple à suivre. Dans la logique des acteurs locaux du tourisme, «les Gorges de la Fou, le site... l'ouvrir au maximum [...] Mieux vaut maintenir une fréquentation un peu soutenue» (Antoine Chrysostome, entretien de 2010). "Pour bien augmenter en fréquentation, il faudrait [...]. On est au maximum sur juillet/ août, maintenant, il faut progresser sur juin et septembre avec des nouvelles clientèles» (employé saisonnier du SIVU des Gorges de la Fou, entretien de 2007).

Effectivement, les variations saisonnières (voir illustration 4) affichent des différences importantes entre les deux mois de pleine saison (juillet et août) et les autres périodes d'ouverture. La moyenne est 24821 visiteurs en juillet, 33659 en août et 29240 en juillet/août. Pour les mois d'avril à mai, la moyenne est de 6179 visiteurs, tandis qu'en septembre et octobre elle est de 6316 . Le site n'ouvre pas toutes les années en octobre et en novembre : en 2009, la fermeture était à minovembre. Bien que le détail des derniers chiffres actuels ne soit pas communiqué, rien n'indique un changement. Ces chiffres de fréquentation des Gorges de la Fou montrent un maximum de 100000 visiteurs au milieu des années 1990 (104 583 visiteurs en 1994) puis une période de travaux avec une baisse relativement brusque (47 635 visiteurs en 1998, en raison de travaux s'étant étalés sur cinq mois lors du début des saisons 1997 et 1998), et actuellement une fluctuation en plateau autour de 75000 visiteurs. En 1999, 79028 personnes ont visité le site, ce qui constitue le minimum atteint en dehors des années de travaux. En moyenne, il y a 86566 visiteurs par an. La courbe supérieure de l'illustration 3 comporte la correction de cette anomalie. Financièrement, le SIVU reverse cette année $15000 €$ à chacune des trois communes d'Arles-sur-Tech, de Montferrer et de Corsavy; une somme très variable selon les années d'investissement sur le site et la présidence du SIVU.

Une étude des différentes plaquettes ou dépliants de présentation (disponibles à l'office de tourisme intercommunal), des communiqués à la presse (Anonyme, 2001; Anonyme, 2007; Anonyme, 2008; Puig, 2009), du site web municipal des Gorges de la Fou, www.ville-arles-sur-tech.fr/gorgesdelafou2. html, puis d'autres ressources internet dans lesquelles une page est consacrée au canyon (Trivago.fr; France This Way.com; Catal66; JeDecouvreLaFrance.com; Visitpedia France) met en évidence une communication centrée sur l'exceptionnalité géologique et géomorphologique du site. Les deux slogans utilisés sont: «Les plus étroites du monde!» et «Un cas géologique unique et étonnant...» (voir illustration 1). Pour atteindre leur clientèle cible, les gestionnaires montrent des familles en situation de découverte du canyon et des roches. Concernant, les filières de commercialisation du site, la démarche reste artisanale. Les Gorges de la Fou ne sont que peu ou pas intégrées 
dans l'offre des grands voyagistes qui proposent des séjours en Catalogne. Le système d'offre (Clary, 1993) reste local. Le président du SIVU explique :

Nos moyens de promotion sont les offices de tourisme, les sites du même genre dans le département [...] les grottes à Villefranche. Avec tous ces sites, on fait échange de prospectus... puis pendant l'été des salariés descendent distribuer le prospectus dans les campings sur toute la côte, jusqu'à Leucate (c'est-à-dire à $80 \mathrm{~km}$ du site) (Antoine Chrysostome, entretien de 2010).

Une étude de fréquentation exploratoire réalisée pendant l'été 2007 (enquête par questionnaire, et trois entretiens avec des employés saisonniers du site au sujet de leurs impressions à propos de la fréquentation), ne permet pas de communiquer précisément des chiffres en raison d'un échantillonnage insuffisant $(\mathrm{N}=142, \mathrm{P}=74281$, où $\mathrm{N}$ est le nombre d'enquêtés et $\mathrm{P}$ est la population). Il est seulement possible de noter que plus de $1 / 2$ des répondants étaient en famille. D'ailleurs, le rapport hommes/femmes ne semble pas déséquilibré. Vraisemblablement, les visiteurs du site s'inscrivent dans la culture des vacances familiales milieu-de-gamme : camping, plage, randonnée, musée ou particularité locale, restaurant et activités sportives pour les enfants. Le taux de fréquentation plus élevé en août qu'en juillet laisse penser à des vacanciers de classes populaires (voir illustration 4). Le public visé dans la démarche de commercialisation du SIVU des Gorges de la Fou semble donc au rendez-vous. Plus surprenant, étant donné la fréquentation internationale du département et la proximité de l'Espagne (ODT Pyrénées-Orientales, 2008; Peypoch et Solonandrasana, 2006), les étrangers représentaient moins de $1 / 3$ des personnes. Enfin, pour une prochaine étude de fréquentation représentative, les variations importantes du tourisme dans la vallée en fonction des saisons obligeront un échantillonnage temporel complet (de début avril à mi-novembre).

Dans les Gorges de la Fou, les visiteurs doivent obligatoirement porter le casque, alors même que l'ensemble du parcours est protégé par un filet métallique à double maille. Aucun passage ne peut être sujet à chutes de pierres (voir illustrations 6 et 7). En fait, la distribution d'un casque comporte plutôt une fonction commerciale et touristique. Premièrement, sur le plan commercial, il donne matérialité au prix du billet (on vous remet un casque, vous n'avez pas l'impression de "payer pour rien»). Deuxièmement, il crée une cohésion identitaire entre les visiteurs, un hexis corporel touristique (tout le monde porte un casque). La similitude vestimentaire rapproche. Plus encore, le casque donne une impression "d'expédition ». Pour les visiteurs, comme par mise en scène, le parcours devient une aventure, mais une aventure en toute sécurité. D’ailleurs, paradoxalement, la sécurité est aussi une attente importante des clientèles en tourisme. Le vacancier ne veut pas risquer sa vie (Chazaud, 2002). En fait, cette grossière mise en scène d'aventure peut satisfaire les visiteurs justement, car ils ne cherchent pas autre chose. Effectivement, quelque soit la non-prise de risque, les Gorges de la Fou représentent bien un territoire du hors-quotidien (Bourdeau, 2003). Selon cette approche, le site satisfait pleinement aux motivations du tourisme, c'est-à-dire le passage d'un ici urbain quotidien à un ailleurs récréatif au sens géoculturel, aussi proche soit-il géographiquement.

\section{Commercialisation touristique, médiation scientifique ou animation culturelle?}

Étant donné leur configuration géomorphologique et leurs propriétés géologiques, les Gorges de la Fou mises en géotourisme offriraient un moyen privilégié de vulgarisation scientifique pour les sciences de la Terre, c'est l'un des principaux enjeux d'une offre en géotourisme (Dowling et Newsome, 2006; Roucan, 1994; Pralong, 2006). L'explication par les géosciences de la formation du site et de son environnement permettrait de reconstituer l'histoire des reliefs en Pyrénées orientales (Calvet, 1996), la formation du Canigou. Les Gorges de la Fou renferment également une flore extrêmement variée, particulièrement intéressante et objet de sorties botaniques pour les associations locales (Anonyme, 2009) qui recensent 83 espèces. On y découvre, par exemple, la Ramonda myconi, aux fleurs tantôt blanches, tantôt violettes, plante endémique du massif pyrénéen, héritière d'un climat chaud pendant le Tertiaire (65 M à 2,6 M d'années), ce qui pourrait être présenté aux enfants comme étant le moment de la disparition des dinosaures (au sujet de la médiation scientifique des notions de temps en géosciences, voir Pralong, 2003). Les Gorges de la Fou et leur flore témoignent aussi des évolutions de température. D'ailleurs les relations entre la Ramonda myconi et le climat font l'objet de recherches scientifiques majeures en botanique (Dubreuil, Riba et Mayol, 2008).

Plusieurs visites à but d'observation participante en 2006, 2007 et 2009, sans que cela soit annoncé ni révélé en fin d'étude, puis des prises de notes, des enregistrements et retranscriptions et 264 photographies permettent de mettre à jour la réalité du travail de médiation scientifique ou simplement d'animation culturelle.

La visite des Gorges de la Fou, dont la durée est estimée à $1 \mathrm{~h} 30$, se réalise sans guide, même sur demande ou réservation. Pour le prix de 6,60 € par adulte, 3,40 € par enfant de 5 à 12 ans et $5 €$ pour les groupes, un casque et une feuille imprimée sont remis. Le document d'une page ne comporte aucun plan, ni schéma ou photographie commentée dans une intention de médiation scientifique. Seul le paragraphe d'introduction comporte les quelques indications suivantes :

Les Canyons de la Fou. Les plus étroits du monde. Sur la route de Prats-de-Mollo, de gigantesques falaises saignées de rouge montent à l'assaut du ciel [...] Le torrent tout menu qui coule sous le pont s'appelle la Fou - d'un vieux mot catalan signifiant précipice car il vient de traverser $2 \mathrm{~km}$ au fond d'une coupure très étroite à travers une montagne de pierre. Et c'est ce dérisoire filet d'eau qui depuis des milliers et des milliers de siècles a scié l'énorme masse du rocher sur une profondeur de 200 à 250 mètres. L'eau a vaincu la pierre et frayé son passage à force d'une obstination, se chiffrant par une somme effrayante de siècles. Ce forage des eaux dans l'énorme bastion de la Fou a été si intense que tout un réseau de galeries et de grottes existe dans son sein et plus encore dans les basses parties du massif calcaire où l'élément liquide poursuit son travail d'érosion (extrait de la feuille-guide de visite). 
Le texte renvoie à la définition d'un canyon, mais plusieurs éléments ne relèvent pas d'une médiation scientifique et restent au niveau d'un argument commercial, avec en particulier l'idée de "canyons les plus étroits du monde» et le caractère de «cas géologique unique et étonnant» que reprend le Guide $d u$ Routard qui estime que les Gorges de la Fou sont «une incroyable curiosité géologique, [...] ces gorges sont les plus étroites du monde» (Josse, 2005). Pourtant le département des Pyrénées-Orientales dispose d'une trentaine de canyons, dont plusieurs avec des encaissements comparables : le Llech, Salt Maria Valenta, le Gourg des Anelles, le canyon du Mas Calsan. On peut aussi regretter : «se chiffrant par une somme effrayante de siècles» plutôt que de situer l'époque par rapport à des repères connus de tous (les dinosaures, la préhistoire...). À l'inverse du document qui annonce «tout un réseau de galeries et de grottes ", les spéléologues que nous avons consultés indiquent un karst restreint non pénétrable, sans résurgence ou émergence repérable (Gabriel Hez, moniteur de spéléologie et président du Club de Spéléologie de Villefranche, géographe et topographe, entretien téléphonique de 2010; Fabrice Fillols, entretien de 2010). Enfin, la dernière phrase du paragraphe suggère une nature sédimentaire de la roche «massif calcaire», mais il n'est pas possible de demander des informations plus précises (alors même que les Gorges de la Fou contiennent du marbre, roche métamorphique résultant de la transformation d'un calcaire). Dans notre cas, les questions obtiennent pour réponse :

- Visiteur (enquêteur) : «Bonjour, pourriez-vous me dire dans quelle roche sont creusées les Gorges?

- Réceptionniste 1 : C'est du calcaire, mais ça, c'est indiqué dans la feuille donnée à l'entrée.

-Visiteur (enquêteur) : ... Euh oui, il est juste marqué «massif calcaire».

- Réceptionniste 2 : C'est du marbre aussi... enfin il y a du marbre, je crois.

-Visiteur (enquêteur) : Mais c'est du marbre ou du calcaire?

[Silence des deux réceptionnistes.]

- Réceptionniste 2 : Enfin c'est sans doute... Il y a plusieurs roches, avec des pierres dans l'eau, c'est une formation géologique naturelle...

-Visiteur (enquêteur) : Mais il y a-t-il un document, une publication, un livre sur les Gorges, et quelqu'un qui pourrait m'expliquer? [note : plusieurs groupes de géologie existent dans le département, et localement, l'association Reynés-patrimoine-culturel organise parfois des sorties géologiques et botaniques dans les Gorges de la Fou].

- Réceptionniste 1 : Non, il n’y pas de guide, mais pourquoi est-ce que vous voulez savoir?

-Visiteur (enquêteur) : Euh... ben, je m'intéresse à la géologie, qui pourrait m'aider?

- Réceptionniste 1 : Ça, on ne sait pas. On ne peut pas vous dire.»

Ainsi, à l'encontre des attentes induites par la communication faite autour du site, la visite des Gorges de la Fou se caractérise par une quasi totale absence de médiation scientifique en géologie, géomorphologie, tectonique, minéralogie, stratigraphie, botanique. Dans le même sens, au sortir de l'étroit du canyon, plutôt que d'inciter à observer l'ouverture assez impressionnante du paysage sur de grandes falaises ou de proposer une table d'orientation, pas moins de six écriteaux affichent «fin de la visite», «téléphone de secours» ou «baignade interdite».

Loin d'un développement autour des géosciences, le contenu de la visite renvoie à un prétendu patrimoine immatériel du lieu, c'est-à-dire plutôt une médiation culturelle. Premièrement, la plus grande partie de la feuille-guide distribuée à l'entrée des Gorges rapporte différentes légendes attachées au lieu : «La légende raconte que ces vertigineux abîmes servaient de résidence...». Le texte détaille la présence de trabucayres cachés au bas des Gorges pour échapper aux recherches des soldats (en catalan, les trabucayres sont des voleurs de grands chemins qui attaquaient les diligences). Les documents et sites internet font référence aux «terribles miquelets», c'est-à-dire les troupes de soldats ou de mercenaires en Catalogne. À chaque fois, un texte qui relève del'animation culturelle. Autre récit, pendant la visite, un panneau indique : «Il y a plus de 50 ans, en cet endroit, une brebis tombée du haut de la falaise fut récupérée vivante par son berger, avec son pelage tout blanc... un vrai miracle...». On remarque l'indication d'un «pelage tout blanc» et la référence explicite au miracle. Deuxièmement, différents passages font l'objet d'un toponyme élaboré pour l'exploitation touristique (voir illustration 5), une dénomination relativement récente, mais légendaire. Des plaques fixées dans la roche en hauteur, complétées quelquefois d'un texte ou d'une installation, annoncent : le Rocher de l'Aigle (un grand aigle découpé en bois peint à été accroché), la grotte des Trabucayres, le Roc $\mathrm{du}$ Soldat, la grotte Saint-Jean (une plaque indique «St Jean Baptiste veille sur vous»), la Petite grotte, la grotte Can Pey. En début de parcours, dans la falaise, une chapelle suspendue contient une sainte Vierge et des cierges avec noté «Nostra Senyora de les canyons de la Fou». La présence religieuse et la mise en place d'une chapelle peut surprendre, d'autant qu'il ne s'agit pas d'installations liées aux prêtres de la paroisse établis dans l'ancienne abbaye d'Arles-sur-Tech (Elie Raubert, curé de la paroisse d'Arles-sur-Tech, entretien de 2010). En fait, la littérature académique montre justement que la mise en tourisme des lieux sombres, étroits ou souterrains s'accommode bien souvent de l'évocation religieuse (Biot, 2006 : 21), comme si la foi chrétienne devait rassurer le public d'un éloignement des enfers qui sont anciennement attachés aux gouffres (Schut, 2006). La sainte patronne des Gorges «vous protège» et tout un ensemble de personnages, trabucayres, soldats... amuse mais ne fait guère peur.

En revanche, une recherche détaillée dans les ouvrages de folklore catalan (Abélanet, 1999; Amades, 1949 et 1982; Blanc, 1979; Chauvet, 1947; Loddo et Pelen, 2001; Roure, 1964; Pere Cerdà, 2008) montre l'existence précise de légendes anciennes qui ne sont pas mentionnées dans les documents ou les sites internet relatifs aux Gorges de la Fou. Autour du Tech et jusqu'aux crêtes frontalières, un vieux berger de la région effrayait tellement les villageois que, par précaution, à sa mort, « on avait cloué son crâne dans le cercueil» pour qu'il ne revienne pas le long du Tech troubler les vivants. Or, quelques anciens racontent avoir retrouvé 

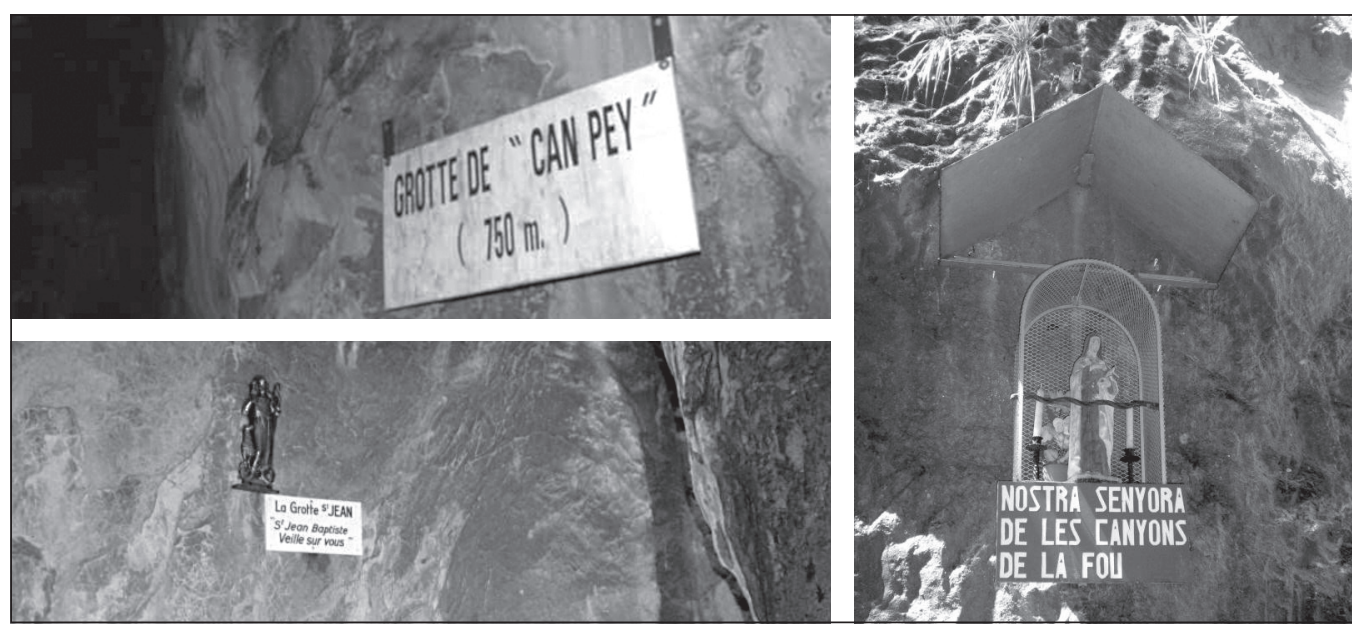

ILLUSTRATION 5 : Toponymes et installations dans le trajet des Gorges de la Fou

(photos : André Suchet). ce clou au bas d'un chemin. Plus précisément, les nuits de pleine lune, au-dessus des Gorges de la Fou, une dame blanche serait visible (Abélanet, 1999 : 149-150). Il s'agirait d'une fée, moitié déesse, moitié sorcière. Enfin, selon plusieurs autres documents, des Dounes d'Aiguat, c'est-à-dire des lavandières nocturnes, se présentent au bas du canyon, dans les vasques d'eau. Parmi les croyances populaires, les lavandières nocturnes appartiennent également à la famille des dames blanches, c'est-à-dire la manifestation sous une forme immatérielle d'une âme défunte qui revient pour expier une faute dont l'origine varie. Elles n'apparaissent qu'aux hommes, les nuits de pleine lune, principalement vers la Toussaint. Elles sont souvent âgées, mais dangereuses pour les vivants. Selon Giraudon (2001 : 66), «elles vivent en groupe [...] Dans les Gorges de la Fou en Roussillon, les Dounes d'aiguat venaient laver leurs draps. Plusieurs légendes évoquent des malheureux qui ont tenté de voler une des pièces de linge [...] dès qu'ils y touchaient leurs bras se pétrifiaient et se brisaient comme du verre».

Il ne s'agit pas ici de déterminer les «vraies» légendes du lieu. Les Gorges de la Fou ont certainement suscité de multiples histoires au cours des siècles au même titre que tous les autres espaces profonds, sombres ou étroits (Schut, 2006), mais l'intention touristique de commercialisation semble ici redéfinir le lieu. Plus qu'une simple sélection patrimoniale (Richard-Bazire et Drouin, à paraître), cet ensemble récent de toponymes ou le remplacement d'une légende de dame blanche en "Nostra Senyora de les canyons de la Fou» (c'està-dire Notre Dame des Canyons de la Fou, rédigé en catalan) paraît rejoindre le principe d'invention de la tradition (Hobsbawm et Ranger, 1983), une invention de la tradition en à peine un demi-siècle motivée par une mise en tourisme. Le travail de valorisation relève seulement d'une animation culturelle (un aigle en bois peint, le récit des miquelets...) ou d'une intention d'animation culturelle (projets de spectacles et d'expositions hors saison).

\section{Tourisme, aménagement et controverse paysagère}

La culture commerçante des acteurs de l'offre en tourisme dans les Gorges de la Fou et la volonté d'une fréquentation maximale expliquent en partie l'aménagement lourd de l'espace (voir illustrations 6 et 7), un aménagement que l'on suppose aussi contraint par une règlementation sécuritaire ou par les exigences des assureurs et les craintes de responsabilités pénales en cas d'accident. Néanmoins, aucun des entretiens avec les responsables du site ne fait directement référence à ces aspects. De plus, les équipements de gorges et canyons aménagés en France sont très divers, les Gorges du Pont du Diable, les Gorges du Fier, Gorges de Kakouetta, celles de la Diosaz... certains des parcours sont très protégés d'autres beaucoup moins. Par exemple, les Gorges de la Fou sont l'un des rares sites entièrement couverts d'un filetauvent de protection. Cet ensemble d'aménagements est sujet à controverse.

La controverse transparaît dans les entretiens de plusieurs visiteurs des Gorges et dans le Guide du Routard qui annonce que les aménagements, avec la passerelle et le filet métallique sécurisent la visite mais "gâchent un peu le spectacle» (Josse, 2005). Dans les commentaires postés sur des sites internet de voyages, un groupe de touristes anglais rédige :

Les Gorges de la Fou sont maintenant une attraction payante, avec un chemin d'accès/passerelle [...] Parfois, vous pouvez à peine voir le haut de la falaise au-dessus, comme de ciseau sur le chemin. Il est une honte, il a été nécessaire de placer une passerelle de métal, filets de protection et les frais généraux, plutôt que d'être autorisés à brouiller le long de la rivière (commentaire posté sur www.fr.francethisway.com/gorgesdelafou.php).

En dépit d'une faiblesse d'écriture en français, le sens se comprend. Le texte vise le non libre accès et l'aménagement. La contestation des visiteurs reste néanmoins marginale. Une recherche sur le site internet Trivago.fr, l'un des principaux comparateurs de prix en tourisme pour l'Europe (chambres d'hôtel et attractions touristiques) donne $90 \%$ de satisfaction et un rapport qualité/prix de $100 \%$. Les quelques critiques récurrentes caractérisent un site touristique : "C'est magnifique, c'est une très belle sortie. Peut-être la seule chose, c'est qu'ils n'ont pas assez de bancs, sur les aires de repos, on était debout.» "Non vraiment très bien, pas de point négatif, enfin si sur le parking, c'était...» (extraits d'entretiens avec les 


\section{ILLUSTRATION 6 : Des visiteurs circulent sur les 1500 mètres de passerelle installés, un filet métallique, visible dans le coin supérieur gauche \\ de la photographie, protège l'ensemble du parcours d'éventuelles chutes de pierres (photo : André Suchet).}

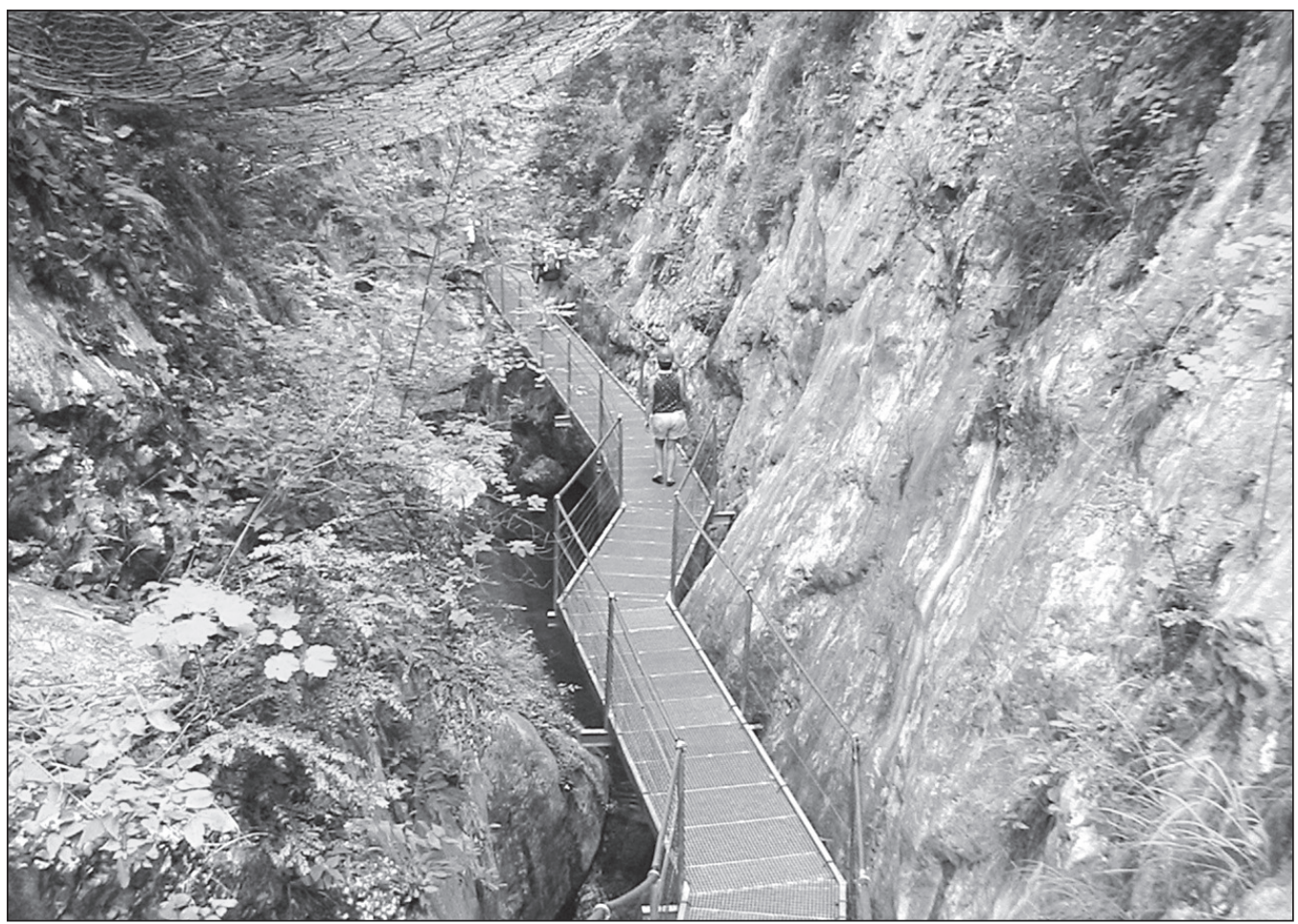

visiteurs, en étant explicitement en situation d'enquête). «Un seul petit point négatif : quelques petits «embouteillages» sur l'étroite passerelle. Quand l'affluence est trop forte, il devient difficile de se croiser ou de se doubler» (commentaire posté sur www.trivago.fr).

L'opposition vient plutôt des non-visiteurs ayant déjà «vu l'aménagement» ou des personnes extérieures au système local des acteurs du tourisme, tout particulièrement des pratiquants experts de canyoning. Plusieurs d'entre eux ont déjà parcouru le canyon en "passant les grillages» durant la période de fermeture. Selon eux, l'aménagement réalisé dans les Gorges de la Fou constitue une dégradation importante de l'environnement. En fait, sous l'apparence et le vocabulaire d'une controverse environnementale, ces discours concernent plus exactement une controverse paysagère. C'est la vue intérieure du canyon qui est en cause. D'ailleurs, à l'inverse, les personnes n'ayant pas déjà connu le site de visu ou par des photographies sont "étonnés de la question", dans leur idée «des sites aménagés, il y en a beaucoup». Ainsi, un nombre important de ces usagers non-visiteurs ayant découvert les passerelles, escaliers, poutrelles et pylônes expriment un sentiment de rejet.

C'est une horreur à mes yeux... Horrible, ça dénature complètement [...] aménager un canyon comme ça, je l'ai jamais vu ailleurs, ni en France... ni... pourtant j'ai fait un peu du canyon aux quatre coins du monde (Gabriel Hez, entretien de 2010).

Les Gorges de la Fou, c'est affreux, tout a été bousillé [...] on dirait qu'on est dans la Tour Eiffel!

Avoir fait ça dans un canyon, c'est un massacre! C'est une honte! Y'a du béton, des piliers, tout est en grillage... la falaise est percée avec des poutres en fer, plus tous les pylônes pour maintenir leurs pare-pierres... [...]. Dans un site comme ça...

C'est vraiment moche, c'est un gâchis monstrueux. Pour faire ça, faut avoir vraiment des dollars à la place des yeux, comme l'oncle Picsou dans Walt Disney. C'est un massacre environnemental, sur un plan écologique, c'est inadmissible (extraits d'entretiens avec des pratiquants experts de canyoning dans le département des Pyrénées-Orientales).

Il faut dire que les responsables des Gorges de la Fou ne sont pas très convaincants sur le sujet d'un développement durable du tourisme et d'une prise en compte de la nature paysagère du canyon. Le secrétaire du SIVU donne pour réponse à la question des impacts environnementaux de l'exploitation touristique : "Il ne peut y avoir aucun problème, les gens n'ont pas tellement accès aux parois (pour dégrader la flore ou la roche). Euh... et pour la faune, c'est très fréquenté, donc ils n'ont pas le temps de faire des déjections dans la rivière» (Claude Couchevellou, entretetien de 2010). L’idée de nature semble assez confuse dans l'idée du secrétaire lorsqu'il indique : "On a fini les travaux de mise en sécurité... On a redonné une nouvelle vie au décor [«le décor» désigne la nature]... De toute façon c'est un site naturel, ça ne risque pas...» Pour un autre responsable, «c'est pas la grotte de Lascaux... le public, il ne risque pas de dégrader! Il y a de l'air au-dessus!». Dans tous les entretiens avec des personnes en charge du site, excepté un, l'impact des aménagements en eux-mêmes n'est pas mentionné. Dans tous les cas, l'aménagement est uniquement associé à la sécurité. Sur plusieurs entretiens le vocable "sécurité» 


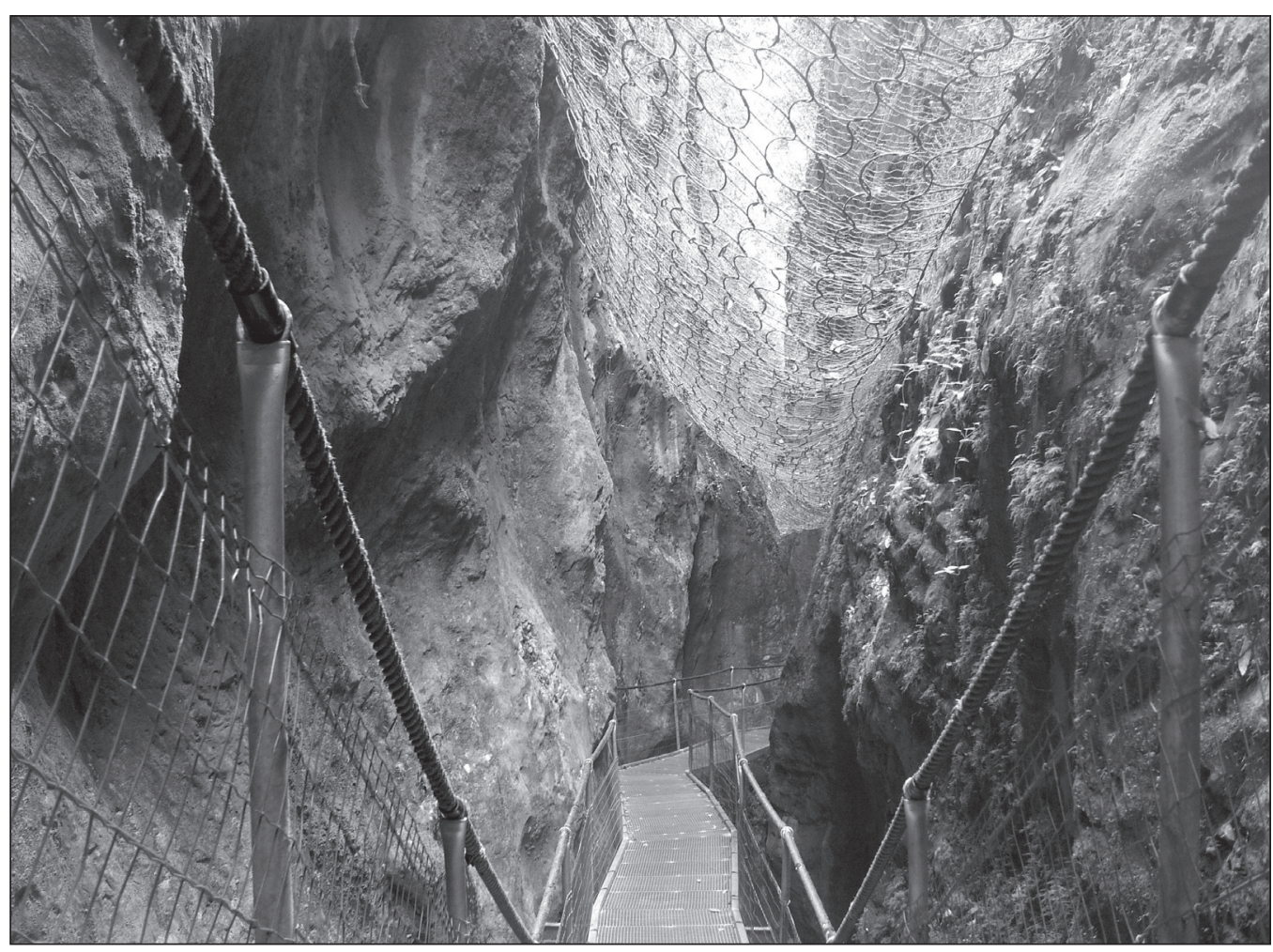

\section{ILLUSTRATION 7 :}

Un aménagement lourd

de l'espace (escalier, passerelle, filet-auvent métallique)

(photo : André Suchet). atteint plus de huit occurrences. "En vous engageant, en toute sécurité, dans les méandres de la Fou» (extrait de la feuille-guide de visite), "la visite est complètement sécurisée, c'est une découverte en toute sécurité... Il y a un auvent, vous êtes en sécurité» (employé saisonnier du SIVU des Gorges de la Fou en conversation avec un groupe de vacanciers sur le parc de stationnement, 2007). Toutefois, cette (sur)protection n'est pas reliée à une justification réglementaire ou d'assurance et responsabilité.

Il ne faut pas caricaturer la position du SIVU des Gorges de la Fou. Un seul des entretiens auprès des responsables du site aborde directement le sujet d'un impact paysager, mais il annonce aussi clairement l'intention «éducative» et sociale du site. Il s'agit de l'actuel président du SIVU des Gorges de la Fou, Antoine Chrysostome, qui débute l'entretien que nous avons eu par : "On fait ce qu'on peut, pour qu'on n'abîme pas trop... ce n'est pas trop joli à l'œil, mais ça permet de le montrer aux gens». Effectivement, au-delà d'un intérêt financier pour les communes, l'exploitation touristique du site repose directement sur le projet d'une démocratisation de la montagne. Tous les entretiens s'accordent à ce sujet :

On s'inscrit je crois dans l'idée des fondateurs [...] On a un site exceptionnel, envie de le montrer... de l'ouvrir au maximum de monde. Des tarifs abordables [...] pour que toute la famille puisse... (Antoine Chrysostome, entretien de 2010)

Le site permet de découvrir les Gorges en toute sécurité. C'est un public familial à qui on offre la possibilité... là c'est tout le monde qui peut le voir [...] beaucoup les enfants, ça a vraiment une visée éducative (il conviendrait de parler plutôt de découverte, que de visée éducative justement au regard de la non-médiation scientifique).

Ça permet à des gens qui sont pas sportifs de voir ces gorges, des gens qui ne les verraient jamais autrement (différents extraits d'entretiens avec des responsables du site, 2007 et 2008).

D’un autre côté, plusieurs des opposants à l'aménagement des Gorges de la Fou (extraits d'entretiens de 2010) reconnaissent : «Les communes, elles ont fait le bon choix, pour elles.» «Puis ça doit bien avoir un impact sur les communes... il faut bien nourrir l'être humain, [...] ça fait vivre les communes.» «Ça crée des emplois dans la vallée, c'est comme une station de ski...»Et le champ de la controverse se complexifie avec les spéléologues ou autres amateurs de nature qui pensent les sites aménagés en tant que moyens de canaliser la fréquentation touristique et donc de protéger d'autres lieux. Un exemple de raisonnement :

Les Gorges de la Fou aménagées [...] Je suis pour...

Il en faut pour canaliser la grande majorité des gens

[...] Je préfère qu'ils aillent dans les Gorges de la Fou, s'il faut sacrifier des sites [...] Je préfère concentrer les personnes sur un site, plutôt que de les répartir dans dix sites où ils vont tout saccager [...] C'est comme une cavité aménagée [référence au tourisme souterrain dans la grotte des Grandes Canalettes à proximité], il en faut (Jean-Louis Perez, président du Conflent Spéléo Club, entretien de 2010).

Par suite, on peut chercher à s'interroger dans quelle mesure les observations réalisées tiennent d'un débat de 
valeurs ou d'un simple conflit d'usage (Mounet, 1996; Perrin et Mounet, 2006) ? Sur l'ensemble des données retranscrites, un peu des deux coexiste en proportions différentes selon les personnes. "C'est affreux à voir... ça gâche tout le site » en appelle à des valeurs, tandis que «c'est un massacre cet aménagement, moi je suis guide, je vous parle ....par rapport au canyon du Llech dans lequel on encadre, avec une grosse fréquentation, pourtant le site n'est pas transformé [...] il aurait fallu laisser le site nature » relève probablement du conflit d'usage avec des raisons économiques et professionnelles. Néanmoins, sur un plan théorique, l'un rejoint l'autre dans la mesure où, à travers un débat de valeurs sur le paysage, se joue une concurrence de perceptions culturelles de la définition légitime de l'espace (au sens de Bourdieu, 1994). Les perceptions devenant constructions (Debarbieux, 2004), c'est ainsi une concurrence de territoires qui resurgit (Di Méo, 1998; et voir l'étude de Greffier, 2002). Autrement dit, dans la controverse paysagère des Gorges de la Fou, les multiples propos d'un débat de valeur relèvent d'une concurrence de territorialisation, et les conflits d'usage sont une concurrence entre territoires déjà présents.

\section{Conclusion}

La présente étude relative aux Gorges de la Fou, en Pyrénées orientales, met en évidence les éléments d'un système local en jeu (Chadefaud, 1988; Clary, 1993) dans la production d'un site touristique (au sens de la typologie du MIT). Il s'agit d'un ensemble d'acteurs de culture commerçante, lié ensuite à la municipalité de trois communes rurales et montagnardes : Arles-sur-Tech, Corsavy et Montferrer. Au départ, l'importance accordée au lieu interroge la possibilité d'une patrimonialisation de cet étroit paysage et de l'environnement du cours d'eau mais, au regard de cette étude, le site des Gorges de la Fou semble plutôt constituer une ressource territoriale en vallée du Tech, c'està-dire une spécificité locale activée par un projet (Gumuchian et Pecqueur, 2007). Un décalage s'observe entre la mise en avant de dimensions géologiques et géomorphologiques « uniques au monde» dans les stratégies de commercialisation du site et la place effective des géosciences dans la découverte du lieu. Confusion entre géologie et géomorphologie, imprécisions quant à la nature des roches, aucune possibilité de visite guidée, les responsables et animateurs des Gorges de la Fou ne semblent pas compétents ni préoccupés des sciences de la Terre. Malgré une fréquentation importante du parcours aménagé, 75000 à 100000 visiteurs par an, les Gorges de la Fou ne contribuent pas vraiment à une diffusion de la connaissance produite en sciences de la Terre.

En revanche, un ensemble de toponymes récents mais légendaires emmène les visiteurs sous la protection de la sainte patronne des Gorges et de saint Jean Baptiste, une présence religieuse en contradiction avec la légende maléfique du lieu. La subversion du lieu est totale : les usages sportifs (canyoning, spéléologie), traditionnels (pêche, chasse) ou scientifiques (excepté quelques sorties d'associations locales) sont écartés. La situation des visiteurs surprotégés d'un casque alors que des filets métalliques recouvrent le trajet met en scène l'aventure, mais une aventure en toute sécurité. Dans tous les cas, il s'agit bien d'un moment de tourisme hors-quotidien (Bourdeau, 2003). Enfin, l'ensemble des passerelles, escaliers, filets métalliques et poutrelles représente un aménagement lourd de l'espace, objet de controverse : une controverse paysagère.

Pour aller plus loin et développer une littérature relative aux gorges et canyons mis en (géo)tourisme, tout comme un grand nombre d'études traite du tourisme souterrain (Nicod, 1998; Gauchon, 1997; Biot, 2006; Biot et Gauchon, 2005), il conviendrait de multiplier les études de cas. On pense notamment aux Gorges du Pont du Diable, aux Gorges du Fier et à celles de la Diosaz dans les Alpes du Nord ou également aux Gorges de Kakuetta en Pyrénées occidentales. Certains de ces sites ont su aménager leur parcours au prix d'un moindre impact paysager, d'autres ont mieux valorisé les connaissances issues des sciences de la Terre, ou peut-être aussi les formes de commercialisation sont différentes. Un travail qui permettrait de mieux connaître cette branche du géotourisme et les possibilités qu'elle représente pour établir encore des liens entre environnement, science et société.

\section{Documents sources}

ANONYME (s. d.) sans titre, <http://www.ville-arles-sur-tech.fr/gorgesdelafou2.htm>, consulté le 20 juillet 2010.

ANONYME (s. d.) «Montferrer : les gorges de la Fou», <http:// catal66.free.fr/vis_virt_po/montferrer-gorgesfou.htm $>$, consulté le 20 juillet 2010 .

ANONYME (2001) «Travel, Forum, Been there...», The Daily Telegraph, 6 janvier.

ANONYME (2007) «Mystère et richesse des eaux souterraines du Vallespir», L'Indépendant, 24 décembre.

ANONYME (2008) «Aujourd'hui Arles-sur-Tech : Visite des Gorges de la Fou », L'Indépendant, 24 juillet.

ANONYME (2009a) «Sortie botanique du Pas de l'Avet à Cortsaví », L'Indépendant, 5 novembre.

ANONYME (2009b) Les Canyons de la Fou, Feuille-guide de visite du site, juillet.

ANONYME (2009c) Les Gorges de la Fou : Les plus étroites du monde, dépliant publicitaire, juillet.

FRANCE THIS WAY (2006-2010) «Gorges de la Fou », France This Way, $<$ http://www.fr.francethisway.com/gorgesdelafou.php>, consulté le 20 juillet 2010.

JE DÉCOUVRE LA FRANCE (s. d.) «JeDécouvreLaFrance.com », Je découvre la France/JDLF, <http://www.jedecouvrelafrance.com/f-1910. pyrenees-orientales-gorges-la-fou.html>, consulté le 20 juillet 2010.

JOSSE, Pierre (responsable) (2005) Languedoc-Roussillon. Le Guide du routard 2005-2006, Paris : Hachette.

PALAU, Paul; Bernard RIEU et Patrice TEISSEIRE-DUFOUR (2009) Canigó. Magie d'une montagne, Le Boulou : Éditions Objectif Sud.

PUIG, Estelle (2009) «Arles-sur-Tech : à l'antre du diable la curiosité naturelle l'emporte », Guide de l'été Midi Libre L'indépendant, p. 83.

TRIVAGO (2004-2010) «Gorges de la Fou », trivago v4_11_4, <http:// www.trivago.fr/arles-sur-tech-103333/randonnee-en-montagneescalade/les-gorges-de-la-fou-154869>, consulté le 22 juillet 2010.

VISITPEDIA (2007-2010) «Gorges de la Fou : Arles-sur-Tech», Sequoia Media, <http://france.vadelis.fr/Mediterranee/Pyrenees_Orientales/ Arles_Sur_Tech/Gorges_De_La_Fou/>, consulté le 20 juillet 2010. 


\section{Références}

ABÉLANET, Jean (1999) Lieux et légendes du Roussillon et des Pyrénées catalanes, Canet-en-Roussillon : Llibres del Trabucaire. 202 p.

AMADES, Joan (1949) El Pirineu : tradicions i llegendes, Barcelona : La Llumenera. $167 \mathrm{p}$.

AMADES, Joan (1982) Folklore de Catalunya I: Rondallistica, Barcelona : Editorial Selecta. $1204 \mathrm{p}$.

BIOT, Vincent et Christophe GAUCHON (2005) «État des lieux du tourisme souterrain en France : la fin d'un cycle?», Karstologia, nº 45-46, p. 41-54.

BIOT, Vincent (2006) «Le tourisme souterrain en France», numéro monographique de Karstologia Mémoires, $\mathrm{n}^{\circ} 15.236$ p.

BLANC, Dominique (dir.) (1979) Récits et contes populaires de Catalogne I: Dans le Vallespir. Paris : Gallimard. 189 p.

BOURDEAU, Philippe (2003) «Territoires du hors-quotidien : Une géographie culturelle du rapport à l'ailleurs dans les sociétés urbaines contemporaines. Le cas du tourisme sportif de montagne et de nature». Mémoire du diplôme d'Habilitation à diriger des recherches, Grenoble : Université de Grenoble 1.267 p.

BOURDIEU, Pierre (1994) Raisons pratiques, sur la théorie de l'action, Paris : Seuil. 251 p.

CALVET, Marc (1996) Morphogenèse d'une montagne méditerranéenne: les Pyrénées orientales, Perpignan/Orléans : Presses universitaires de Perpignan/BRGM. 3 volumes.

CARRERAS-MARTI, Joan et Jesús GIRALT-RADIGALES (dir.) (1993) Diccionari de la llengua Catalana, Barcelona : Enciclopèdia Catalana.

CHADEFAUD, Michel (1988) Aux origines du tourisme dans les pays de l'Adour. Du mythe à l'espace : un essai de géographie historique, Pau : Publication de l'Université de Pau et des Pays de l'Adour. 1010 p.

CHAUVET, Horace (1947) Traditions populaires $d u$ Roussillon, Perpignan : Imprimerie du Midi. $247 \mathrm{p}$.

CHAZAUD, Pierre (2002) «L'offre de sécurité entre développement durable et risque zéro ", Cahier Espaces, nº 73, p. 135-140.

CHOPPY, Jacques (1985) Dictionnaire de spéléologie physique et karstologie. Édition de l'auteur. 148 p.

CLARY, Daniel (1993) Le tourisme dans l'espace français, Paris : Masson. $358 \mathrm{p}$.

DEBARBIEUX, Bernard (2004) «De l'objet spatial à l'effet géographique», DANS L'effet géographique, sous la direction de Bernard DEBARBIEUX et Marie-Christine FOURNY, p. 11-33. Grenoble : Maison des Sciences de l'Homme des Alpes.

DI MÉO, Guy (1998) Géographie sociale et territoires, Paris : Nathan. 317 p.

DOWLING, Ross Kingston et David NEWSOME (dir.) (2006) Geotourism, Amsterdam : Elsevier. 260 p.

DUBREUIL, Marta; Miquel RIBA et Maria MAYOL (2008) «Genetic structure and diversity in Ramonda myconi (Gesneriaceae): effects of historical climate change on a preglacial relict species", American Journal of Botany, vol. 95, $\mathrm{n}^{\circ}$ 5, p. 577-587.

DUVAL, Mélanie (2008) «Patrimonialisation et mise en tourisme des espaces naturels : l'exemple des gorges de l'Ardèche», Géographie et Cultures, $\mathrm{n}^{\circ}$ 66, p. 61-78.

GAUCHON, Christophe (1996) «Friches touristiques : les anciennes grottes touristiques en France", DANS Actes du Symposium international Grottes aménagées et contrôle de l'environnement souterrain, Frabosa Soprana, p. 11-20.

GAUCHON, Christophe (1997) «Des cavernes et des hommes. Géographie souterraine des montagnes françaises ", numéro monographique de Karstologia Mémoires, ${ }^{\circ}$ 7. 248 p.
GIRAUDON, Daniel (2001) «La lavandière de nuit Ar gannerez-noz», DANS Êtres fantastiques des régions de France, sous la direction de Daniel LODDO et Jean-Noël PELEN, p. 45-78. Paris : L'Harmattan.

GREFFIER, Luc (2002) «La descente de canyon, des territoires en concurrence», Sud-Ouest Européen, no 13, p. 77-84.

GUMUCHIAN, Hervé et Bernard PECQUEUR (dir.) (2007) La ressource territoriale, Paris : Anthropos. $252 \mathrm{p}$.

HOBSBAWM, Eric et Terence RANGER (dir.) (1983) The Invention of Tradition, Cambridge : Cambridge University Press. 320 p.

KNAFOU, Rémy; Mireille BRUSTON; Florence DEPREST; Philippe DUHAMEL; Jean-Christophe GAY et Isabelle SACAREAU (1997) «Une approche géographique du tourisme», L'Espace Géographique, vol. 26, n 3, p. 193-203.

LAZZAROTTI, Olivier (2003) «Patrimoine», DANS Dictionnaire de la géographie, sous la direction de Jacques LEVY et Michel LUSSAULT, p. 692-693. Paris : Belin.

LEMARTINEL, Bertrand (1998) Les «Orgues» d'Ille : de la beauté à la science, Perpignan : Publication UPRES-A 6042 CNRS. 12 p.

LODDO, Daniel et Jean-Noël PELEN (dir.) (2001) Êtres fantastiques des régions de France, Paris : L'Harmattan. 304 p.

MIT (2002) Tourismes 1 : Lieux communs, Paris: Belin. 320 p.

MOUNET, Jean-Pierre (1996) «Sports d'eau vive et pêche en rivière : un conflit asymétrique», STAPS, vol. 17, n 40, p. 7-21.

NICOD, Jean (1998) «Les grottes : rétrospective historique et insertion des grottes-aménagées dans l'espace géographique», Annales de Géographie, vol. $107, \mathrm{n}^{\circ} 603$, p. 508-530.

ODT Pyrénées-Orientales (2008) «La fréquentation touristique des PO», Comité départemental du tourisme Pyrénées-Orientales, $<$ http://observatoire-cdt66.typepad.fr/frequentation/>, consulté le 12 septembre 2010.

PALMER, Arthur N. (2007) Cave Geology, Dayton, OH : Cave Books. 454 p.

PERE CERDĀ, Jordi (2008) «Dames d'eau en Pyrénées catalanes», Mythologie française, vol. 58, n 230, p. 23-28.

PERRIN, Clémence et Jean-Pierre MOUNET (2006) «L’organisation de la pratique du canyoning sur un site : le canyon du Furon", Science \& Motricité, $\mathrm{n}^{\circ} 57$, p. $79-103$.

PEYPOCH, Nicolas et Bernardin SOLONANDRASANA (2006) «Panorama et analyse descriptive de la fréquentation touristique européenne en Languedoc-Roussillon», Téoros, vol. 25, nº 1, p. 50-54.

PRALONG, Jean-Pierre (2003) «Valorisation et vulgarisation des sciences de la Terre : les concepts de temps et d'espace et leur application à la randonnée pédestre", Travaux et recherches de l'Institut de géographie de l'Université de Lausanne, $\mathrm{n}^{\circ}$ 24, p. 115-127.

PRALONG, Jean-Pierre (2006) «Géotourisme et utilisation de sites naturels d'intérêt pour les sciences de la Terre : les régions de Crans-Montana-Sierre (Valais, Alpes suisses) et Chamonix-Mont-Blanc (Haute-Savoie, Alpes françaises) ». Thèse de doctorat, Lausanne : Université de Lausanne. 224 p.

REYNARD, Emmanuel (2005) "Géomorphosites et paysages», Géomorphologie, vol. 11, nº 3, p. 181-188.

RICHARD-BAZIRE, Anne et Martin DROUIN (dir.) (à paraitre) La sélection patrimoniale. 6 e rencontre internationale des jeunes chercheurs en patrimoine.

ROUCAN, Jean-Pierre (1994) «Vulgariser les sciences de la Terre», Géologues, $\mathrm{n}^{\circ} 102$, p. $42-48$.

ROURE, Nicole (1964) «La sorcellerie en Roussillon ». Thèse de $3^{e}$ cycle, Montpellier : Université de Montpellier. 133 p.

SCHUT, Pierre-Olaf (2006) «Les usages des grottes au fil du temps», Géographie et Cultures, n 57, p. 103-122.

SCHUT, Pierre-Olaf (2007) L'exploration souterraine. Une histoire culturelle de la spéléologie. Paris : L’Harmattan. 394 p. 